\title{
Identification and Reconstruction of Michel Electrons in ProtoDUNE-SP
}

Aleena Rafique

On behalf of the DUNE Collaboration

Argonne

\section{Motivations}

- Key demonstration for DUNE electron selection and energy reconstruction and to demonstrate DUNE far detector capability to identify and reconstruct low-energy electron events.

- The analysis is important to show that ProtoDUNE can use the topological / calorimetric information provided by the TPC to identify a specific topology.

\section{DUNE/ProtoDUNE Experiment}

\section{DUNE:}

- $1300 \mathrm{~km}$ baseline

70 kton Liquid Argon Time Projection Chamber (LArTPC) Far

Detector (FD) at SURF, South Dakota, $1.5 \mathrm{~km}$ underground

- Multiple technologies for the Near Detector (ND) at Fermilab

- Will measure neutrino oscillation probability to determine mass

ordering and CP violation phase; potential for BSM physics and supernova neutrinos

ProtoDUNE-single phase:

- $\sim 7 \times 6 \times 7 \mathrm{~m}^{3}$ in charged test beam at CERN

A crucial part of the DUNE effort towards the construction of the

first DUNE far detector module

ProtoDUNE-SP I operated from September 2018 to July 2020
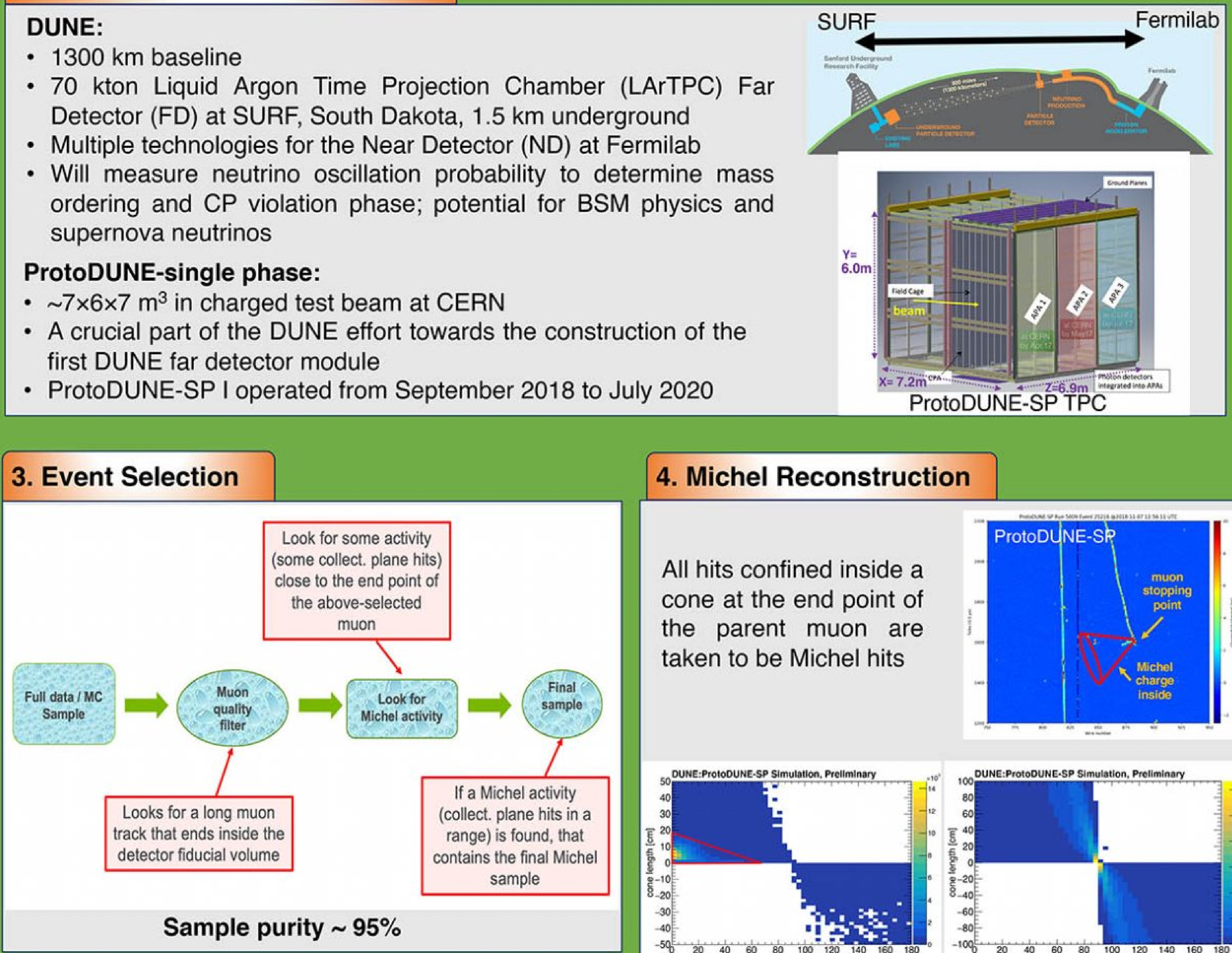

4. Michel Reconstruction

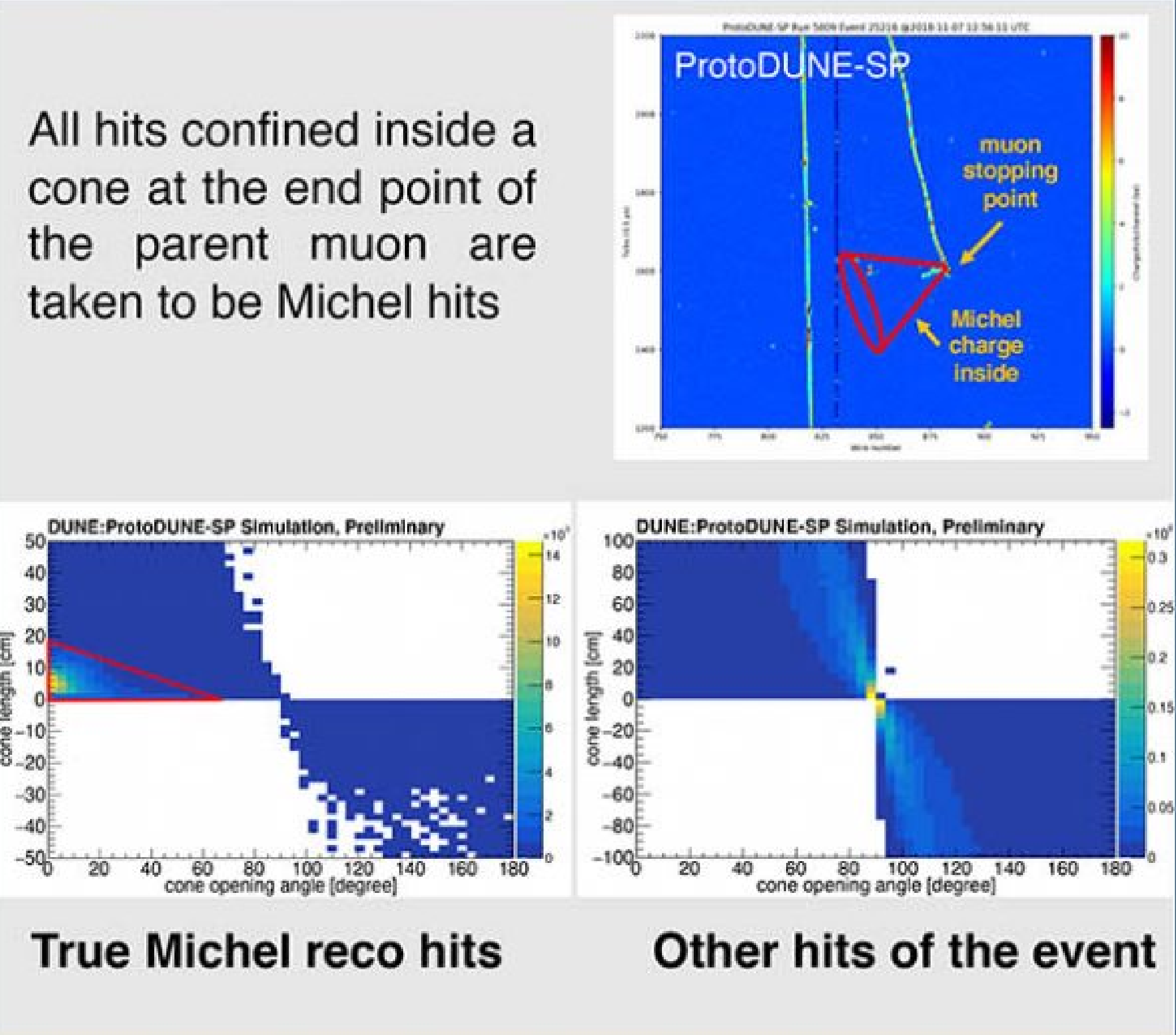

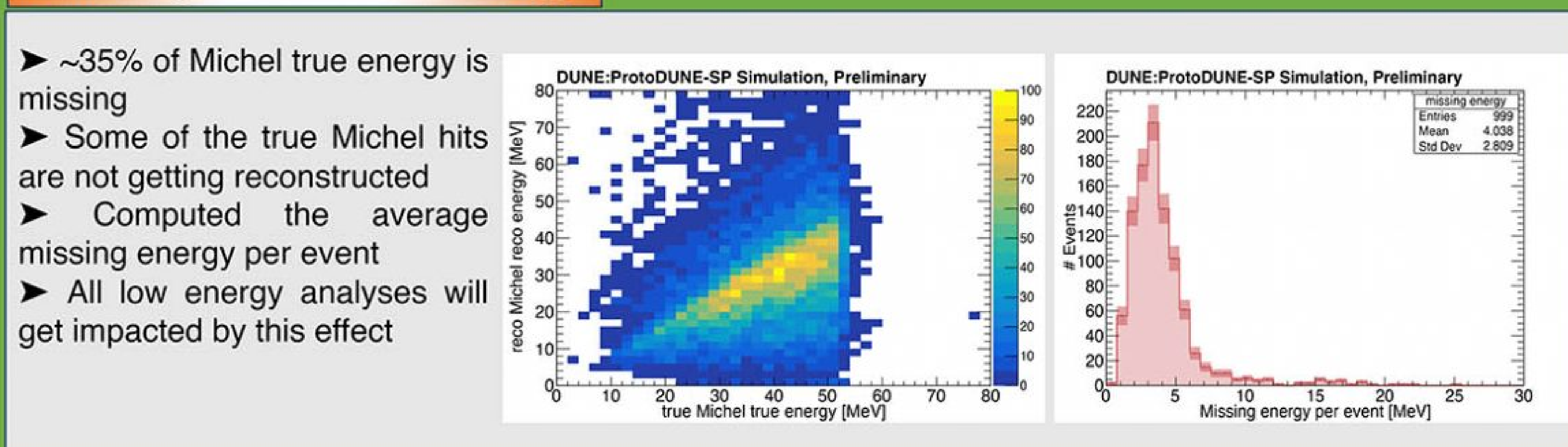

\section{Updated MC Michel Energy}

- MC Michel reconstructable energy after the addition of the $M C$ missing energy per event and the true Michel energy spectrum are compared

$>11 \%$ energy due to hit reconstruction threshold effects was retrieved using MC studies - The energy resolution improves
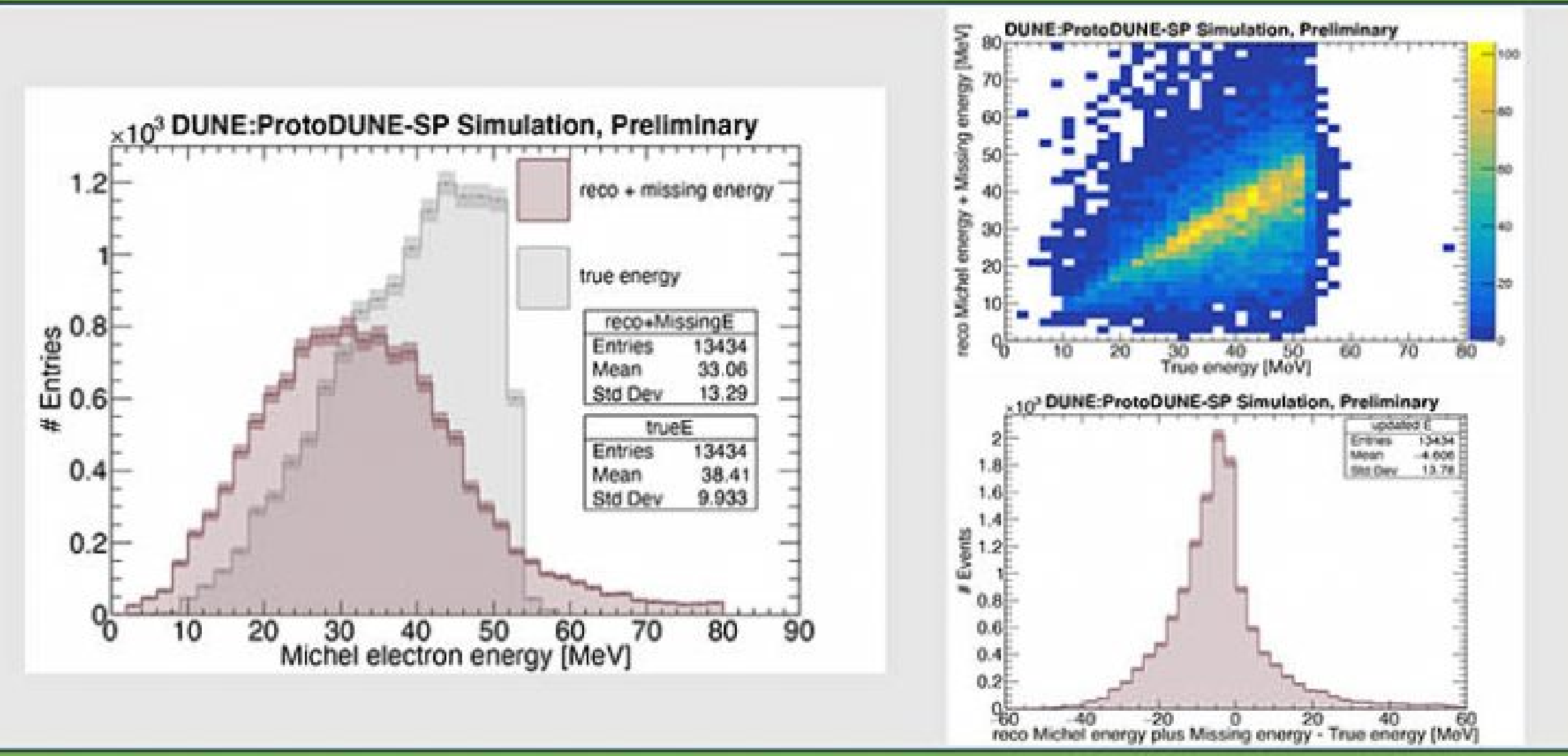

7. Michel Reconstructed Energy

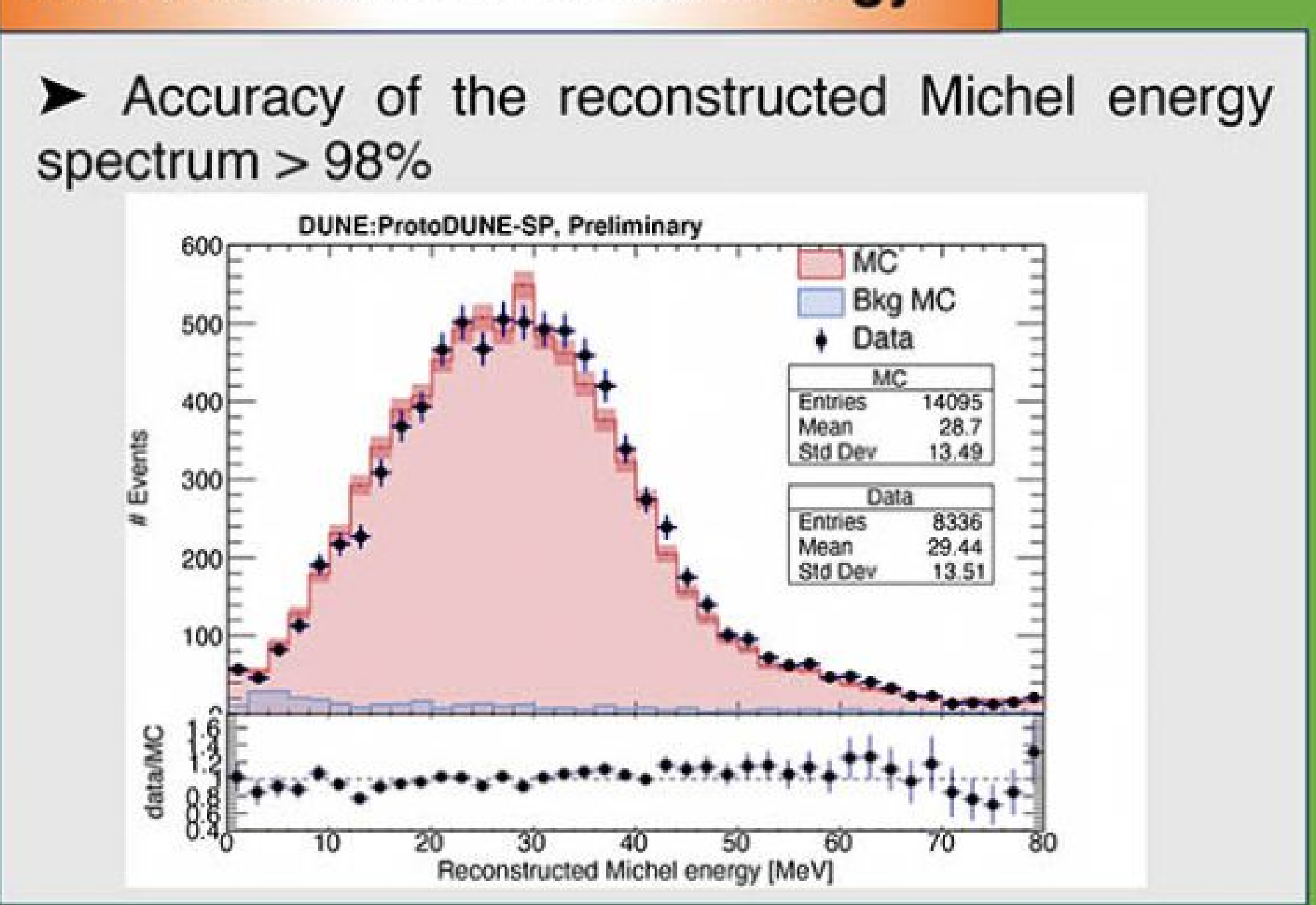

8. Conclusions and Future Direction

- Developed Michel selection, reconstruction, and and energy cal
DUNE LArTPCs.

-- Achieved $95 \%$ event purity

Michel electron energy accuracy is $>98 \%$

Working on understanding the remaining source of missing energy

The energy outside Michel cone is another main reason of the energy loss

Michel analysis in ProtoDUNE-SP paper is in progress 